\title{
Tecnología y sociedad: La evaluación de impacto social del Plan Ceibal
}

\author{
(1) Martínez, A.
}

Contacto: amartinez@plan.ceibal.edu.uy

(1) Plan Ceibal. Coordinación de Evaluación de Impacto Social del Plan Ceibal, Laboratorio Tecnológico del Uruguay (LATU)

Recibido: 16/10/2008 - Aprobado: 18/11/2008

\section{$\underline{\text { Resumen }}$}

El objetivo del presente artículo es realizar una breve presentación del Plan Ceibal y de la estrategia de evaluación de su impacto social (EIS) que se lleva a cabo desde el LATU. En la primera parte se presenta el Plan Ceibal, sus orígenes y objetivos, así como información básica sobre su implementación. En segundo lugar se reflexiona brevemente sobre la relación entre tecnología e inclusión social. El tercer punto es una presentación del diseño general de investigación para la evaluación del Plan.

\section{Abstract}

The goal of this article is to briefly present the Plan Ceibal and the Social Impact Evaluation (SIE) strategy undertaken at the LATU. The first section introduces the origins and objectives of the plan, as well as basic information on its implementation. The second is a short reflection on the relationship between technology and social inclusion. The third section shows a general presentation of the research design for the evaluation.

\section{El Plan Ceibal}

El Plan CEIBAL ("Conectividad Educativa de Informática Básica para el aprendizaje en Línea", siendo el ceibal el campo de árboles de ceibo, la flor nacional del Uruguay), es pionero a nivel mundial en entregar una laptop a cada niño y docente de las escuelas públicas del país y brindar conexión inalámbrica a Internet en cada centro escolar. Además de la provisión de tecnología, supone acciones de capacitación, apoyo, evaluación y seguimiento.

El Plan Ceibal busca promover la inclusión digital con el fin de disminuir la brecha existente entre ciudadanos uruguayos, así como respecto a otros países, de manera de posibilitar un mayor y mejor acceso a la educación y a la cultura.

Se diferencia de esfuerzos anteriores llevados a cabo en que su objetivo no se limita a dotar a los centros educativos de equipamiento y accesibilidad, sino que la herramienta llega al hogar a través de los alumnos. El proyecto en general apunta a garantizar el uso de la herramienta, prevé la formación docente, la elaboración de contenidos y la promoción de la participación familiar y social.

En concreto, el proyecto supone dotar de un computador personal a cada niño y a cada maestro, así como brindar al colectivo docente la capacitación, los materiales, las orientaciones y el apoyo necesarios. Los escolares y docentes beneficiarios del plan en todo el país, así como sus familiares, podrán 
trabajar con la computadora en sus domicilios o en el aula, en forma individual o en red con otras personas.

Como contexto del Plan cabe mencionar que a partir de 2005 se crea en el país una nueva institucionalidad vinculada a los temas y necesidades de la así llamada Sociedad de la Información y el Conocimiento. En esta institucionalidad destacan la Agencia para el Gobierno de Gestión Electrónica y Sociedad de la Información y el Conocimiento (AGESIC) y la Agencia Nacional de Investigación e Innovación (ANII). Tanto la Agenda Digital Uruguay 2008 - 2010 como el Plan Estratégico de la ANII se orientan hacia el desarrollo, la innovación y la inclusión social. Es en estas estrategias que se inscribe el Plan CEIBAL.

Es importante mencionar que en Uruguay existía una amplia red de acceso a servicios de telecomunicaciones -en particular en términos comparativos respecto al contexto latinoamericano- que el Plan amplía considerablemente, pero cuya existencia es importante para su viabilidad.

El Plan Ceibal comenzó a implementarse como tal en octubre de 2007, creándose a través de un Decreto Presidencial. Inicialmente se implementó el Proyecto Ceibal como piloto en una localidad del interior del Uruguay, Villa Cardal, en mayo de 2007. Luego de una instancia de evaluación, se resolvió su generalización a nivel del país.

A la fecha (octubre de 2008) se han entregado 126.000 computadoras portátiles a docentes y niños pertenecientes a más de 900 localidades del interior del país. Se proyecta completar la entrega en el interior en 2008 y realizar la entrega en la capital y el área Metropolitana en 2009, completando de esta forma el total del país. La población total beneficiada en forma directa por el programa ascenderá a 340.000 niños y docentes. Dado que los escolares trasladan la laptop diariamente a sus hogares hay, además, más de un millón de beneficiarios potenciales no directos.

El Plan Ceibal involucra a una multiplicidad de organismos y allí radica una de sus originalidades y fortalezas. El decreto presidencial que lo creó define que la toma de decisiones en torno al Plan está a cargo de una Comisión Política integrada por representantes de organismos públicos vinculados a la educación, la innovación y la tecnología. Esta comisión está integrada por delegados de: la Presidencia de la República, la ANEP (Administración Nacional de Educación Pública), el Consejo de Educación Primaria, el Ministerio de Educación y Cultura, ANTEL (Administración Nacional de Telecomunicaciones), la AGESIC (Agencia para el Desarrollo del Gobierno Electrónico y la Sociedad de la información), la ANII (Agencia Nacional de Investigación e Innovación) y el LATU (Laboratorio Tecnológico del Uruguay), que tiene a su cargo la implementación del Plan. Esta Comisión tiene como cometido acordar las condiciones, ritmos y contenidos para llevar a cabo el Plan. Además, funciona una Comisión de Educación que tiene a su cargo la elaboración de contenidos, la sensibilización a docentes y directivos, y el portal educativo vinculado a Ceibal.

En cuanto a las características de las laptops entregadas, vale describir brevemente que están especialmente diseñadas para el uso por parte de niños, y esto incluye tanto a su hardware (pequeño, resistente a golpes) como a su software (con contenidos y fines educativos, con una interfase especialmente concebida para niños). Los escolares la trasladan a su hogar además de usarla en la escuela, y pueden conectase entre sí a través de una red llamada "Mesh". En sus hogares también pueden conectarse a Internet utilizando la expansión de la señal que se emite desde el centro escolar, en ocasiones a través de otra laptop, que la expande. En este sentido, dado que una computadora sirve de "puente" de conexión a otras, los hogares algo más alejados del centro escolar también pueden acceder a la señal. La meta en este sentido es que ningún niño deba caminar más de 300 metros para tener conectividad. Por otra parte, se dispone de servicio wi-fi en algunas plazas públicas del país, con lo cual también se puede hacer uso de la laptop en los espacios públicos.

Otra característica de esta tecnología, que tiene un impacto social potencial interesante, es que facilita la construcción de redes. Cuando una laptop está encendida se pueden visualizar en la pantalla todas las laptops de la red Ceibal que están conectadas, y de esta forma comunicarse con ellas, promoviendo el trabajo colaborativo y la comunicación interpersonal. Este es un aspecto importante a nivel local; sin embargo, la conexión con el mundo es mucho más impactante, pues permite a los niños y familiares interactuar con otras personas a nivel mundial. En este plano, también el acceso a fuentes de información y conocimiento se ven potenciados, y su impacto probablemente será más fuerte cuanto menor el nivel socioeconómico de partida. En otras palabras, se espera que aquellos ciudadanos que disponían de menor acceso a fuentes de información y conocimiento sean los más beneficiados. 


\section{Tecnología, innovación e inclusión social}

Es relevante destacar que el Plan Ceibal se crea como una estrategia de desarrollo para la inclusión social. La mejora en la plataforma de infraestructura y de conectividad que está generando constituye la base material de cualquier iniciativa de inclusión social a través de las TIC. La literatura sobre el tema reconoce este elemento como un factor imprescindible, pero no suficiente, para lograr la inclusión social.

Este es un punto importante que se refleja en algunos de los objetivos del Plan Ceibal. En concreto, el Plan no consiste simplemente en la entrega de laptops, sino que incluye la capacitación y producción de contenidos a través de la escuela primaria pública y el apoyo a la ciudadanía para el mejor aprovechamiento de la herramienta. Se apunta de esta forma no solo a innovar en los procesos educativos, sino a proveer una base social para una inclusión digital significativa y equitativa.

En el mundo contemporáneo, las TIC han facilitado la difusión de los conocimientos e información en volúmenes y velocidades sin precedentes en la historia de la humanidad. Sin embargo, las nuevas tecnologías y sus potencialidades han sido principalmente explotadas en el terreno financiero y empresarial, y su incorporación en los procesos de desarrollo social es aún deficiente (Castells, 2001, entre otros).

En este marco se habla de "brecha digital", concepto que remite fundamentalmente a la desigualdad en el acceso a las TIC. Esta brecha existe entre países y entre regiones dentro de un mismo país. La concepción de brecha digital restringida al acceso implicó que las acciones y políticas de algunos países hicieran énfasis en la dinámica de los mercados y en las estrategias de regulación de las TIC, o implementando políticas centradas en la ampliación del acceso a ellas. No obstante, dadas las desigualdades estructurales existentes en las sociedades, en particular las latinoamericanas, resulta imprescindible acompañar la ampliación del acceso con medidas que apunten al aumento de las capacidades de los ciudadanos para beneficiarse del uso de las nuevas tecnologías y lograr su real democratización (Warschauer, 2003; Rueda Ortiz, 2005).

Siguiendo los aportes de la literatura sobre este tema, resulta necesario evaluar los impactos del acceso a las nuevas tecnologías en relación a las variables que muestran la estratificación de la población, y considerar su pertinencia de acuerdo a las necesidades y características de los ciudadanos. Un concepto que resulta útil para considerar la multidimensionalidad del problema y que se busca incluir en la evaluación es el de "inclusión digital" (Finquelievich, 2003; Warschauer, 2003). La operacionalización de este concepto permite considerar las características y necesidades de los sujetos. Un concepto estrechamente ligado al de inclusión digital es el de uso con sentido. Este tipo de uso implica conocer los medios (en particular, pero no solo, Internet), saber cuándo y cuáles usar al servicio de objetivos individuales o colectivos. Resulta claro que este uso está condicionado por la capacidad y las posibilidades de los sujetos para ser productores de contenidos, así como de su acceso a información y conocimiento que les resulten útiles (Camacho, 2001).

En cuanto al Plan y sus objetivos, el aporte al aumento de las capacidades de los ciudadanos se intenta operativizar a través de acciones de apoyo al uso en coordinación con otras políticas estatales, organizaciones de la sociedad civil y universidades. Se apunta a estimular el uso de la laptop, sensibilizar a la población y hacer circular la información entre los nuevos usuarios, muchos de los cuales nunca han tenido contacto con una computadora. Probablemente el más interesante y potente de estos colectivos es la Red de Apoyo al Plan CEIBAL (RAP Ceibal), que moviliza a unos 700 voluntarios y que ha contribuido a la capacitación y apropiación del plan por parte de la población y los docentes.

En suma, el Plan Ceibal se posiciona como una política de innovación articulada con la inclusión social, combinación que no es frecuente, y desde allí debe ser evaluada.

\section{La Evaluación de Impacto Social (EIS)}

Un elemento vital para cualquier política es realizar el seguimiento y la evaluación de los resultados de las acciones implementadas, de forma tal que se puedan superar obstáculos y profundizar en los procesos positivos que resultan de ella. En el marco del Plan se implementan mecanismos de 
seguimiento y evaluación de las distintas líneas de trabajo y de sus resultados, y se procura realizar una atribución de impacto al Plan, que si bien está limitada en su diseño por el avanzado nivel de implementación del mismo (al momento prácticamente todo el interior del país), pueda proveer de conocimientos válidos en torno a esta política. Algunos de los rasgos más relevantes de la evaluación se describen a continuación.

Las principales dimensiones a evaluar se desprenden de la formulación de los objetivos del Plan. No obstante, el diseño de evaluación no está solo centrado en objetivos, sino que considera y prevé el estudio de otros posibles impactos identificados.

De acuerdo a los objetivos del Plan (www.ceibal.edu.uy y Decreto Presidencial del 18/10/2007), dotar en forma universal a los escolares uruguayos de una herramienta informática que facilite la conectividad a través de la educación pública permitirá a los escolares y a sus familias acceder, apropiarse y producir conocimiento y participar de nuevas ideas, con el objetivo de generar impactos positivos en:

- La disminución de la brecha digital a nivel nacional.

- El acceso a través del escolar de los demás integrantes de su familia a los servicios informáticos globales, sin importar localidad geográfica ni condición social.

- Los modos de vincularse de todos los ciudadanos con la información y el conocimiento, con la consiguiente ampliación del acceso a nuevos servicios y mejores oportunidades laborales.

- El aumento de aportes originales e innovación en diversos niveles, del país en el contexto internacional, como resultado de esta masificación del uso de las NTICs.

- La mejora en los procesos de enseñanza y de aprendizaje, el aumento de la motivación para vincularse con el conocimiento en niños y maestros y la efectiva incorporación de la alfabetización informática.

En otras palabras, el principal foco de la evaluación son los resultados del Plan Ceibal en la disminución de la inequidad social a través de la implementación de medidas que apuntan a universalizar el acceso y el uso de las nuevas tecnologías a nivel nacional. Estos resultados pueden estudiarse diferencialmente en niños, hogares, escuelas y comunidades. En forma complementaria, este trabajo articulará los resultados del estudio de los procesos de enseñanza y aprendizaje que fue encomendado a la Evaluación Educativa del Plan Ceibal con sede en ANEP.

La evaluación combina un carácter exploratorio y descriptivo en una primera instancia, y en la segunda etapa procurará elaborar explicaciones de los resultados hallados.

Se investigará principalmente en qué medida el Plan Ceibal contribuye a la reducción de la brecha digital y a la promoción de la inclusión digital. En este plano, se toma a la brecha digital como un problema multidimensional, relacionado con otras brechas sociales, económicas, geográficas, etarias y de género. Para ello se propone relevar no solo el acceso, sino el uso y los sentidos relacionados con la posesión de una laptop en la población beneficiaria del Plan. Finalmente, apunta a relevar experiencias innovadoras y buenas prácticas asociadas a su implementación.

Una característica de este abordaje es que si bien se potenciarán todos los aportes internacionales con que se cuenta al momento, dado el carácter pionero de la experiencia en Uruguay no se cuenta con investigaciones de referencia de casos comparables. La segunda característica es que la evaluación realizada desde el LATU se encargará de generar una parte de la información, mientras que otros capítulos del informe se elaborarán en coordinación con otros investigadores e instituciones que se encuentran abocadas al estudio del Plan Ceibal, funcionando en los hechos como parte de un sistema de evaluación del Plan.

\section{Objetivos de la investigación-evaluación}

\section{Función diagnóstica:}

- Elaborar una línea de base con indicadores que permitan un seguimiento a futuro de los impactos del Plan en la esfera social, cultural, económica y de participación democrática. 
- Identificación y caracterización de los actores y grupos más relevantes, en particular en lo relativo a estratificación por Nivel Socio-económico y relación previa con las TIC.

- Analizar cómo varían a través del tiempo el nivel y calidad del uso de la herramienta y la participación social en la producción de contenidos.

\section{Evaluación de procesos:}

- Generar un flujo de información periódico en torno a las tres principales componentes de la implementación del plan: capacitación, distribución y conservación de la laptop, y su uso.

- Relevar las acciones de capacitación y apoyo realizadas desde el Plan Ceibal y desde la sociedad civil, así como las coordinaciones interinstitucionales potenciadas por el Plan Ceibal.

- A partir del análisis de la información, realizar recomendaciones para la toma de decisiones.

\section{Evaluación de resultados e impactos:}

- Conocer el nivel de manejo de la herramienta por parte de los niños (más allá de su uso escolar) y su impacto en la autoestima, en la predisposición a innovar, en las aptitudes y actitudes vinculadas al trabajo colaborativo y en red, analizado según el contexto sociocultural y las habilidades de partida. Conocer el uso que los familiares y niños hacen de esta tecnología fuera del entorno escolar y el proceso de apropiación que realizan con respecto a la nueva herramienta.

- Estudiar en qué medida y bajo qué condiciones la nueva herramienta comienza a ser utilizada a nivel de las comunidades; con qué objetivos, qué acciones están teniendo mayores efectos positivos y qué necesidades se detectan.

- Conocer los cambios en las oportunidades, comportamientos, conocimientos, posibilidades, percepciones y bienestar de los integrantes de los hogares y comunidades destinatarios del Plan Ceibal, y los factores que contribuyen a ello así como los que lo obstaculizan.

- Analizar los cambios en los vínculos y relaciones al interior de la familia y los cambios en las prácticas sociales y en el uso del tiempo libre que se producen luego de la introducción de la laptop en el entorno social del niño.

- Analizar la participación en redes de las personas, estudiando el impacto atribuible a la concepción colaborativa de la laptop de Ceibal.

- Estudiar el impacto en la autoestima de los niños, en su motivación para aprender y en las oportunidades disponibles para ellos.

- Realizar un seguimiento de las iniciativas de desarrollo social y productivo local que se sirven de la red Ceibal.

\section{Diseño de evaluación y metodologías}

\section{El diseño}

El diseño combina tres estrategias complementarias, hace uso de metodologías cuantitativas y cualitativas, y apela a fuentes de información tanto primarias como secundarias, en línea con las recomendaciones internacionales sobre este punto. 
a) Evaluación pre-post intervención. Se basa en una observación inicial antes de la implementación del Programa (Línea Base) y en sucesivas observaciones en el tiempo post intervención en una muestra de escuelas de Montevideo y Canelones.

Como estrategia complementaria:

b) Comparación de escuelas que participan en el Proyecto CEIBAL teniendo diferentes tiempos de implementación del Plan. Las escuelas de similares características con poco (o nulo) tiempo de implementación funcionan como grupo testigo. Se realizan mediciones anuales en las escuelas con diferente tiempo de implementación y se comparan indicadores seleccionados, permitiendo analizar en qué medida sus variaciones en el tiempo pueden ser atribuidas al impacto del Proyecto o, por el contrario, podrían derivar de factores externos.

c) Realización de estudios de casos de interés seleccionados. En los estudios de casos, seleccionados intencionalmente, se podrán observar en mayor profundidad las dinámicas comunitarias, familiares y con respecto al centro escolar, y extraer aprendizajes.

\section{Metodologías y técnicas}

a) Análisis de información estadística nacional. Para el relevamiento de los cambios en la brecha digital en el acceso y uso de computadoras e Internet se plantea analizar la Encuesta Nacional Continua de Hogares Ampliada 2006 (ECHA-2006) del Instituto Nacional de Estadística (INE) en el plano nacional. Para relevar información en torno a la brecha digital internacional se plantea analizar información proveniente de organismos internacionales principalmente OSILAC (de CEPAL).

b) Realización de encuesta a niños, docentes y familiares de los niños. Encuesta en Centros de la RED RUTELCO sobre el perfil de usuarios, cambios y nuevas necesidades detectadas, identificación de sinergias con Ceibal.

c) Entrevistas con padres, maestros, directores, actores locales clave e informantes calificados.

d) Observación del entorno comunitario y del uso de las laptops.

e) Monitoreo remoto del uso de las laptops, accediendo a la información de una muestra de servidores escolares sobre tiempo de uso según grado escolar y contexto social de la escuela.

f) Análisis de información del servicio de atención telefónica de Ceibal.

g) Registro de las actividades de capacitación e información destinadas a la comunidad (en coordinación con RAP).

A la fecha, el proceso de evaluación se encuentra en etapa de trabajo de campo, en la cual se han realizado visitas a centros escolares y entrevistas exploratorias a maestros, niños y padres, así como el pilotaje de los instrumentos de encuesta. En estas instancias se tuvo una aproximación a algunos resultados sugerentes en términos de acceso, uso y apropiación de la nueva herramienta -en particular por parte de los niños-, así como a los desafíos pendientes en términos de apoyo a la ciudadanía y capacitación docente para asegurar que el proceso sea exitoso. A comienzos de 2009 se contará con datos representativos a nivel nacional complementados con información cualitativa detallada sobre los primeros resultados del Plan Ceibal.

\section{Referencias}

- CAMACHO JIMÉNEZ, Kemly. The Internet: a tool for social change? Elements of a necessary discussion [En línea]. Costa Rica: Fundación Acceso, 2001, [Consulta: 21 de octubre de 2008]. Disponible en: http://www.acceso.or.cr/sites/acceso.or.cr/files/20010830-Camacho-InternetandSocialChange.pdf

- CASTELLS, Manuel. Tecnología de la información y capitalismo global. En: Hutton, Will; Giddens, Anthony (ed.). En el límite: la vida en el capitalismo global. Barcelona: Tusquets, 2001. p. 81-112 
- FINQUELIEVICH, Susana. Innovación, información y prácticas sociales. En: Universidad de Antioquía, et al. Primer Congreso Internacional de Investigación en Ciencias de la Información, (Medellín 8-10 de noviembre de 2006). Medellín: UdeA, 2006.

- IAIA. Principios internacionales de la evaluación de impacto social [En línea]. Fargo: IAIA, 2004, [Consulta: 21 de octubre de 2008]. (Serie Publicaciones Especiales; 2). Disponible en: http://www.preval.org/documentos/00426.pdf

- RUEDA ORTIZ, Rocío. Apropiación social de las tecnologías de información: ciberciudadanías emergentes. En: Tecnología y Comunicación Educativas. 2005, (41):19- 32.

- WARSCHAUER, Mark. Technology and social inclusion: rethinking the digital divide. London: MIT, 2003.

\section{Páginas web}

Plan Ceibal. Disponible en: http://www.ceibal.edu.uy

Rap Ceibal. Disponible en: http://rapCEIBAL.blogspot.com 